\title{
The Application and Prospect of Informative Facility in Hospital Infect Management
}

\author{
Zhao Shuliang, Liang Dingcai \\ School of Public Administration \\ Yunnan University of Finance and Economics \\ Kunming, P. R. China \\ pingyuanzhaosan@163.com
}

\author{
Cui Li \\ Department of Health Research \\ Yunnan health and development research association \\ Kunming, P. R. China \\ 95105274@qq.com
}

\begin{abstract}
Medical institution improving the infection information management system to improve the management level of hospital infection. Play to the advantages of the network and manage hospital infection. Further improving update and increase the hospital environmental microbiology monitoring computer management system, surgical procedures, the infection rate of the medical system and the hospital infection monitoring and warning system is based on the hospital infection cases of computer management system. Hospital infection information management can have a purpose, a focus, to carry out monitoring work to avoid the blind investment funds, save money by the collection of dynamic information.
\end{abstract}

Keywords-Informative facility, Hospital, infect Management.

At present, the hospital infection monitoring work is extensive in the national hospital. Monitoring method from past review the survey has gradually transited to the prospective investigation. And the hospital infection outbreak should be timely detected and, early investigated to take timely measures to cut off the chain of transmission, controlling the outbreaks. According to these requirements, hospital infection monitoring, disinfection ,sterilization ,the hygienic monitoring, antibacterial drugs using monitoring ,surveillance of bacterial resistance, hospital infection management quality etc. information system and the hospital infection management website has carried out the comprehensive development of the hospital infection management related to forward-looking monitoring, real-time and monthly for statistical data analysis, reporting, feedback, and to achieve management and public information sharing, improve the management level of hospital infection and departments make concerted efforts, received good results. Practice has proved that the conclusion and information management of hospital infection is inevitable trend for further development of hospital infection management.

\section{THE APPLICATION OF INFORMATION TECHNOLOGY IN THE MANAGEMENT OF MEDICAL INSTITUTIONS}

\section{A. Monitoring of special drug resistant strains}

With the rapid development of medical technology. The disease especially in critically ill treatment has constantly improved is mostly based on the wide application of broad- spectrum antibiotics is one of the important means, however. The abuse of antibiotics in clinical treatment is a very common phenomenon. But the strong pressure of antibiotics Inevitably produced a large number of drug-resistant strains. These strains become a thorny problem in today's hospital infection and the difficulty of the treatment has increasingly become an important problem. So. The monitoring of these resistant strains is more important. (gold (gold (the software in nosocomial infection pathogens and drug resistance spectrum of new increased the resistance category column, including MRSA resistant methicillin-resistant Staphylococcus aureus); MRSE (resistant to methicillin resistant Staphylococcus aureus); mrcns resistant methicillin resistant coagulase negative staphylococci); VRSA (vancomycin resistant Staphylococcus aureus), VRE (vancomycin resistant enterococci); PRSP (resistant to penicillin Streptococcus pneumoniae); CRPA (resistant to ceftazidime Pseudomonas aeruginosa bacteria); ESBL producing extended spectrum beta lactam enzyme of gram negative bacteria) and other types of computer according to the entry of the microbiological laboratory susceptibility report result automatic judgment.

\section{B. Hospital infection epidemic, outbreak warning system}

Hospital infection is usually sporadic, sometimes it can be an outbreak. Hospital infection prevalence of $2 \% .4 \% .1$ million people hospitalized patients with nosocomial infection can have a 1 outbreak epidemic. Survey of CHIP (total hospital infection program) was found. Even if the hospital has a very active surveillance system, there are still 3, 1 of the gathering of the infection could not be identified: that has never occurred in the hospital. The purpose of hospital infection is to control and prevent the occurrence of nosocomial infection, especially to prevent or reduce the occurrence of nosocomial infection. There is still not a very reliable monitoring system to alert the outbreak or epidemic of nosocomial infections. In order to find out the epidemic trend of nosocomial infection in a more timely fashion. (2) the function and characteristics of the early warning subsystem. Prospective surveillance of nosocomial infection is one of the effective methods to discover and identify the epidemic or outbreak of nosocomial infection in a timely manner. Once found infections aggregation phenomenon. The early warning system will be automatically prompted to infection of full-time staff in epidemiological survey was conducted before. To analysis and 
identify whether there is a true infection outbreak or epidemic. In the case of hospital infection, the hospital infection was monitored at the time of the hospital infection. Computers will automatically enter the warning window. If the preset range is more than or equal to 3 cases of hospital infection cases and drug resistant strains. The interface will show the relevant patient information. To play the role of warning; users can also according to the actual requirements set up their own warning period (days of early warning can set their own 3 days, 7 days or 10 days, visual monitoring target), early warning value (for 3 cases. Can also be white line set) after the implementation of the update command. System automatically press department pathogen infection, the infection sites, pathogens infection cases, infection sites of infection cases of four part respectively statistics show is more than or equal to 3 cases of hospital infection cases and drug resistant strains. Using the hospital infection information dynamic. Is key to carry out monitoring work. So as to give full play to the role of forward-looking information in the early warning. As long as the full time analysis of early warning content, it is possible to find out the problem as soon as possible, to take measures to prevent or block the outbreak or epidemic.

\section{Information management of hospital infection in surgical operation}

Objective to investigate the nosocomial infection rate in surgical patients. The surveillance of nosocomial infection is one of the important targets for the development of hospital infection. Can understand the incidence of nosocomial infection, risk factors, and found the problem in time. In order to take timely measures to achieve effective control of infection after the purpose of; at the same time, also calculated the operating surgeon specific infection rate, through timely feedback. Prompted them to find postoperative infection. Take various measures, prevention of hospital infection.

\section{INFORMATION MANAGEMENT OF MEDICAL INSTITUTION INFECTION MANAGEMENT}

How to improve and perfect the management of hospital infection, establish the prevention system of public health emergency in general hospital, which is an important problem to be solved in the development of hospital infection management. How to effectively make use of information system in public health emergencies prevention system of traditional Chinese medicine hospital infection management in the study area for the stand-alone version cannot achieve regional resource sharing defects. Hospital information management in the future will no longer be installed on the user's computer system. And the user does not need to download and install the program. Hospital infection data entry work on the Internet can be. In data entry and summary the monitoring center can be automatic remote backup of computer data. Hospitals, regional and national monitoring center can keep abreast of the hospital infection. Really play its forward-looking function. Hospital infection information management is an inevitable requirement for improving the management level of hospital infection, and is also an effective demand for hospital infection control.

It's extremely meaningful to launch Information management of medical institution infection management actively carry out or participate in Information management of medical institution infection management.

\section{Acknowledgment}

This article was supported by a 2-years' program: Innovation project of Yunnan Provincial Education Department. We also appreciate the support from Yunnan Health and Development Research Association. Thanks also to all the respondents of the research.

\section{References}

[1] Hospital management of patients with acute coronary syndrome: Influence of age, gender and admission department on medical choices and outcome,F Seccareccia, S Rosato, etc. Giornale Italiano Di Cardiologia, 2012, 13.

[2] Discussion on Construction on the Hospital Files Management System under the Informative Condition, $\mathrm{P}$ Zhou,Journal of Linyi University, 2011.

[3] Prescribing habits and caregiver satisfaction with resources for dosing children: Rationale for more informative dosing guidance, JS Barrett, M Narayan, D Patel, Bmc Pediatrics, 2011.

[4] An Empirical Analysis of the Military Hospital's Fixed Asset Management Reform,LI Shu-Yuan $\square$, HU Jing-Bin,Medical \& Pharmaceutical Journal of Peoples Liberation Army, 2012.

[5] G. Eason, B. Noble, and I.N. Sneddon, "On certain integrals of Lipschitz-Hankel type involving products of Bessel functions,” Phil Trans. Roy. Soc. London, vol. A247, pp. 529-551, April 1955. (references)

[6] J. Clerk Maxwell, A Treatise on Electricity and Magnetism, 3rd ed., vol. 2. Oxford: Clarendon, 1892, pp.68-73.

[7] I.S. Jacobs and C.P. Bean, "Fine particles, thin films and exchange anisotropy,” in Magnetism, vol. III, G.T. Rado and H. Suhl, Eds. New York: Academic, 1963, pp. 271-350.

[8] K. Elissa, “Title of paper if known,” unpublished.

[9] R. Nicole, "Title of paper with only first word capitalized," J. Name Stand. Abbrev., in press.

[10] Y. Yorozu, M. Hirano, K. Oka, and Y. Tagawa, "Electron spectroscopy studies on magneto-optical media and plastic substrate interface,” IEEE Transl. J. Magn. Japan, vol. 2, pp. 740-741, August 1987 [Digests 9th Annual Conf. Magnetics Japan, p. 301, 1982].

[11] M. Young, The Technical Writer's Handbook. Mill Valley, CA: University Science, 1989. 